\title{
TRANSICIÓN ENERGÉTICA, INNOVACIÓN Y USOS DIRECTOS DE ENERGÍA GEOTÉRMICA EN MÉXICO: UN ANÁLISIS DE MODELADO TEMÁTICO
}

\author{
Diana Rivera Delgado, ${ }^{a}$ Fernando J. Díaz López ${ }^{\mathrm{b}}$ \\ y Graciela Carrillo González
}

Fecha de recepción: 3 de agosto de 2020. Fecha de aceptación: 26 de enero de 2021.

https://doi.org/10.22201/iiec.20078951e.2021.206.69713

Resumen. En este texto se analiza factores para el desarrollo y la difusión del conocimiento y la innovación para tecnologías asociadas a usos directos de energía geotérmica (UDEG) en México, bajo el contexto de políticas para la transición energética durante el periodo 2008-2019. Se utilizó el enfoque de Sistemas de Innovación Tecnológica (siт) dada su utilidad para identificar los mecanismos que determinan el desarrollo y la difusión de una tecnología emergente en un sistema. El análisis implicó también la elaboración de dos modelos temáticos de Asignación Latente de Dirichlet (ALD), cuyos resultados mostraron que sIT para UDEG en México se encuentra en etapa formativa y responde al motor del empuje de la ciencia y la tecnología.

Palabras clave: Sistema de Innovación Tecnológica; transición energética; usos directos de energía geotérmica; motores de innovación; funciones.

Clasificación JEL: Q42; Q54; Q58.

\section{ENERGY TRANSITION, INNOVATION, AND DIRECT USES OF GEOTHERMAL ENERGY IN MEXICO: A THEMATIC MODELING ANALYSIS}

\begin{abstract}
This article analyzes factors of the development and diffusion of knowledge and innovation for technologies associated with direct uses of geothermal energy (DUGE) in Mexico, within the context of energy transition policies during the period 2008-2019. The Technological Innovation Systems (TIS) approach was employed for this analysis, given its usefulness in identifying the mechanisms that determine the development and diffusion of an emerging technology in a system. The analysis also involved the elaboration of two thematic models of Latent Dirichlet Allocation (LDA), the results of which showed that TIS for DUGE in Mexico is in a formative stage and is driven by science and technology.
\end{abstract}

Key Words: Technology Innovation System; energy transition; direct uses of geothermal energy; innovation drivers; functions.

${ }^{a}$ Universidad Autónoma Metropolitana-Unidad Xochimilco (UAM-x), México; ${ }^{b}$ Technopolis Group, Francia; Innovation for Sustainable Development Network, Francia; Universidad Stellenbosch, Facultad de Ingenieria Industrial, Africa del Sur y SEEDs, Italia. Correos electrónicos: dprd85@outlook. com, fernando.diazlopez@inno4sd.net y gcarri@xoc.uam.mx, respectivamente. 


\section{INTRODUCCIÓN}

En las últimas dos décadas del presente siglo, el estudio de la transición energética y el papel que la innovación y la tecnología tienen en el desarrollo de soluciones para la suficiencia energética y el desarrollo de los países (principalmente las economías emergentes) ha cobrado mayor relevancia en las comunidades de estudios del desarrollo, sistemas de innovación, transiciones a la sostenibilidad, y sistemas energéticos (Sandoval,1998; Santoyo-Castelazo et al., 2014; Vázquez, 2015; Araújo, 2014; Wieczorek, 2018). De acuerdo con la Agencia Internacional de Energías Renovables (IRENA, por sus siglas en inglés, 2017), la transición energética (TE) es el camino para descarbonizar el sistema energético mundial, transformándolo en uno de cero-carbono. Bajo el supuesto que el despliegue acelerado de las energías renovables y la TE impactarían en el desarrollo de los países, sobre todo en las economías emergentes, se asume que el desarrollo de soluciones tecnológicas apropiadas genera un mayor bienestar para la sociedad a través de la creación de empleos y la mejora de la calidad de vida (IEA, 2019). En el proceso de cambio de los sistemas socio-técnicos y con el objetivo de promover la competitividad y la sostenibilidad de las economías nacionales (Coenen y Díaz López, 2010), la innovación es un factor clave para impulsar el proceso de TE a través del desarrollo y difusión de conocimientos para tecnologías de energía renovable, que permite diversificar las aplicaciones más allá de la generación y distribución de energía eléctrica (Araújo, 2014).

Con base en lo anterior, el gobierno mexicano fijó metas de mediano y largo plazo para promover una mayor participación de las energías renovables en la matriz energética, a través de diversos instrumentos de política pública orientados a ese fin. En la Ley de Transición Energética se propuso que 35\% de la generación eléctrica del país sería basada en energías limpias para el 2024 (SENER, 2013).

A nivel internacional la discusión de la TE se centra en analizar el papel de la energía solar y eólica. En años recientes, el tema de la TE basada en tecnologías limpias y sostenibles tiene mayor importancia en México, sobre todo para explorar patrones emergentes en otras áreas como bioenergía, energía oceánica, e incluso el hidrógeno de bajo carbono (Morales et al., 2017; Silva, 2019). Sorprendentemente, para entidades como la Agencia Internacional de Energía (2019), la energía geotérmica se considera una fuente renovable de energía con gran potencial de desarrollo tecnológico, aunque con pocos avances en términos de penetración en la matriz energética global en comparación con otras tecnologías de generación de energía eléctrica basada en renova- 
bles. México tiene gran potencial para el aprovechamiento de la geotermia en diversas manifestaciones, sin embargo, su uso se limita a aplicaciones de recreación (balneología) y tratamientos terapéuticos (Hiriart, 2011; Santoyo et al., 2012).

El presente trabajo centra su atención en analizar el estado de desarrollo del Sistema de Innovación Tecnológica (sIT) para UDEg en México, a través del estudio de sus componentes (actores e instituciones) y propone la inserción de UDEG en el análisis de TE, como una opción que impulsa la diversificación del sector energético con el aprovechamiento de UDEG en procesos industriales, sistemas de enfriamiento para alimentación y almacenes, sistemas de calefacción a nivel distrito, climatización de edificios, secado de alimentos, provisión de energía para invernaderos y acuacultura inteligente, entre otros (Sircar et al., 2016; Lund y Toth, 2020).

De acuerdo con el reporte de Energía Geotérmica para México de la Agencia Internacional de Energía (IEA, 2019), a nivel nacional existe un potencial probado y probable de 1005.8 mw de capacidad instalada para Energía Geotérmica (EG) en 2019. Para en el mismo año, UDEG reporta 156 MW, con un total de calor utilizado de $1162.1 \mathrm{GWH} /$ año distribuidos en más de 160 sitios en 19 estados de la República mexicana (Romo-Jones et al., 2020), estos recursos son principalmente agua caliente con una temperatura promedio de $50^{\circ} \mathrm{C}$ y una capacidad instalada de $155.8 \mathrm{MW}$, lo que representa un fracción marginal de su potencial (Santoyo-Gutiérrez y Torres-Alvarado, 2010).

A nivel internacional una de las aplicaciones de UDEG más comunes es la calefacción de distritos. En países como Islandia y China se utilizan para resolver problemas de bajas temperaturas con una tecnología que permite proporcionar calor constante a bajo costo y con una menor huella de carbono (Tomasini-Montenegro et al., 2017). En otros lugares se ocupa también para fines industriales muy variados; por ejemplo, en Italia UDEG se emplean para fabricar cerveza y leche pasteurizada (Kiruja, 2011; Líndal, 1992; Link et al., 2015; Lund y Boyd, 2015; Ragnarsson, 2003). En la figura 1 se puede observar la evolución de la capacidad instalada de UDEG de acuerdo con su aplicación.

Los UDEG además de generar electricidad pueden generar valor agregado en diversos sectores económicos; en México se tienen documentados un par de casos de empresas nacionales que desarrollaron proyectos de generación de energía eléctrica a partir de geotermia, como los proyectos desarrollados por Grupo Dragón, que en octubre de 2014 instaló una planta geotérmica de operación comercial en San Pedro Lagunillas, Nayarit; en 2018 en vinculación con el grupo Instituto de Ingeniería Desalación y Energías Alternas (iideA) de 


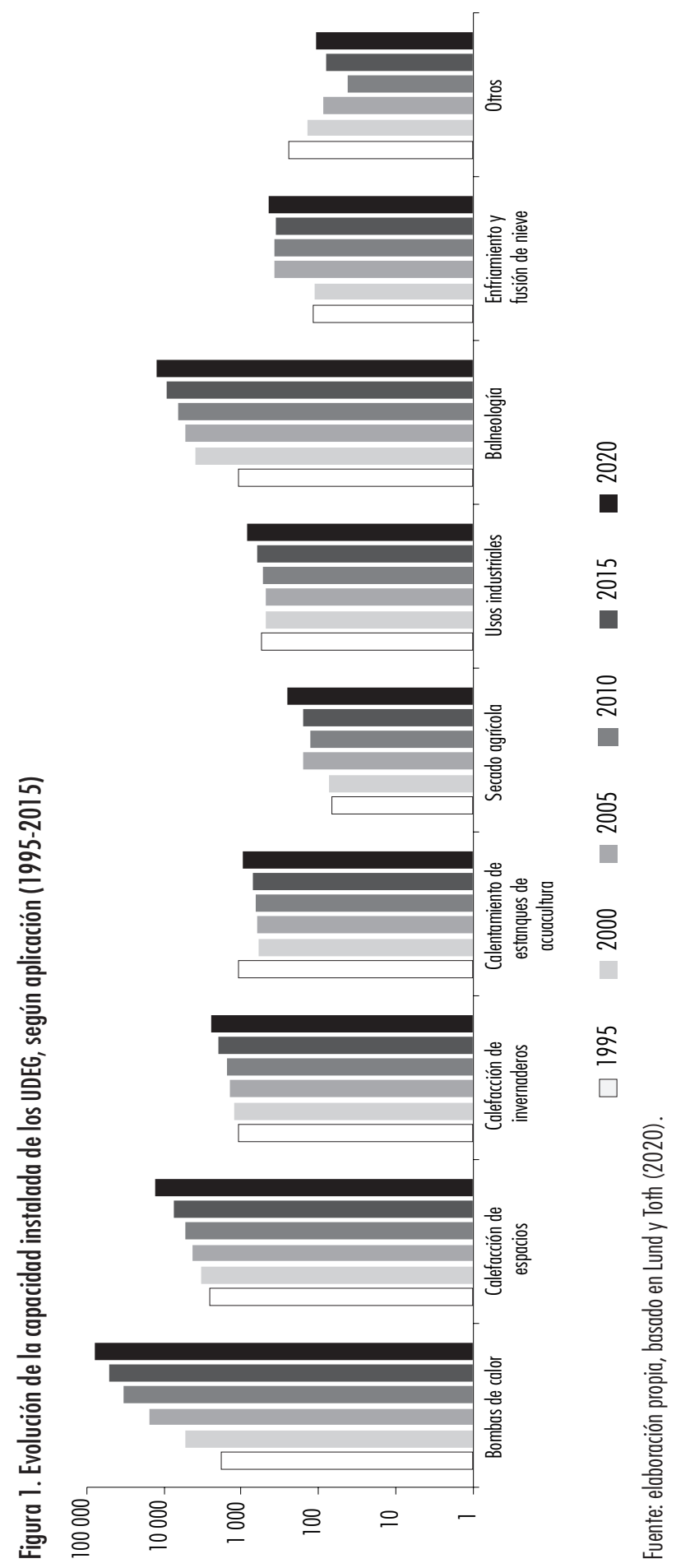


la Universidad Nacional Autónoma de México (UnAm) instaló un Deshidratador Geotérmico de Alimentos que realiza sus procesos a través de UDEG.

Se retoma la literatura de funciones del sit y motores de innovación para analizar la formación de un sistema que promueva el desarrollo de tecnologías para diversas aplicaciones de UDEG. Dicha perspectiva señala que los desarrollos tecnológicos se dan en el contexto de un sistema que se compone por una red de actores, instituciones, tecnologías e interrelaciones que se generan entre ellos (Carlsson et al., 2002). Sin embargo, dado que el sit se forma de manera gradual, las tecnologías emergentes pasarán por una etapa formativa antes de que se pueda esperar que se encuentren en una fase de difusión de mercado (Jacobsson y Bergek, 2004b). El rendimiento de los sit puede ser analizado a través de las llamadas funciones del sistema que responden a diversos motores y se definen con base en las actividades dominantes que participan en él, considerando también el nivel de desarrollo de los componentes. Varios estudios han utilizado el marco del sit para analizar los mecanismos clave que explican la dinámica de los sistemas de innovación y su efecto en el desarrollo y la difusión de la tecnología (Bergek et al., 2008; Markard y Truffer, 2008).

Las preguntas de investigación a desarrollar en el trabajo son las siguientes: ¿cuáles son y cómo se caracterizan las funciones que participan en la formación del SIT de UDEG? y ¿a qué motor de innovación pertenecen las funciones del sit de UDEG? Para darles respuesta, esta investigación desarrolla una modelación teórica de las funciones que participan en la formación de un sistema para tecnologías emergentes y se realizó una estimación empírica utilizando un modelado temático, que es un enfoque totalmente automatizado de análisis de texto.

El análisis automatizado de grandes volúmenes de texto mediante métodos de minería de datos cualitativos (qualitative data mining) es un área de creciente interés en las ciencias políticas y la investigación de políticas públicas con aplicaciones recientes en el área de eco-innovación y transiciones sostenibles (Díaz López y Montalvo, 2015a y 2015b; Coenen y Díaz López, 2010). De acuerdo con Nowlin (2016), este método trata de categorizar y sacar conclusiones de los documentos de manera sistemática, una técnica que facilita esta categorización es el modelado de temas.

El objetivo del presente trabajo fue identificar las funciones existentes en el SIT de UDEG para determinar el grado de desarrollo del sistema y determinar a qué tipo de motor de innovación corresponde su desarrollo. Se utilizó el enfoque de modelado temático (MT) con la aplicación del algoritmo de Asignación Latente de Dirichlet (LDA)para establecer qué funciones se cumplieron en el periodo 2008-2019. Se generaron dos modelos que analizaron los temas 
latentes representativos de las funciones cumplidas en el sit para los componentes del sistema: instituciones y actores.

El artículo se divide en cuatro secciones después de la introducción. En la primera se aborda la discusión teórica de los SIT en etapa formativa, las funciones y los motores de innovación. La segunda hace una contextualización institucional de UDEG en México y las aplicaciones y tecnologías que se desarrollaron en ese periodo. En la tercera se describen método y datos utilizados para el estudio. La cuarta sección muestra los resultados obtenidos y su análisis por componente, así como las conclusiones.

\section{SISTEMAS DE INNOVACIÓN TECNOLÓGICA EN ETAPA FORMATIVA Y MOTORES DE INNOVACIÓN}

De acuerdo con Edsand (2019), el marco del siт puede ser usado para analizar la formación y el crecimiento de los sistemas de innovación. En países emergentes, el análisis tiende a centrarse, en la mayoría de los casos, en la etapa formativa. El enfoque de los sit tiene como objetivo analizar el rendimiento del sistema y los factores que afectan su desempeño (Hekkert et al., 2007), y sus contribuciones teóricas recientes han abonado a la discusión sobre el papel que juega el desarrollo tecnológico con visión sustentable para estimular la transición energética.

Este enfoque se ha utilizado en varios trabajos para analizar energías renovables en países emergentes. Por ejemplo, Tigabu et al. (2015) lo aplican para analizar la aparición de un sit de biogás en Ruanda. Sawulski et al. (2019) estudian cómo se genera un SIT en países "seguidores" de tecnología en el caso de la energía eólica marina en Polonia. Por otro lado, Bento y Fontes (2015) investigan el proceso de construcción de un nuevo sit basado en energía eólica, en el que resaltan la necesidad de mejorar las capacidades de asimilación de las tecnologías para el caso de Portugal; mientras que Perrot (2015) aplica el enfoque de funciones del sit para seguir la evolución de los agentes, redes e instituciones que han influido en la generación y difusión de tecnología solar y eólica en Sudáfrica e India.

\section{Sistema de Innovación Tecnológica}

La literatura del sit destaca que las tecnologías pasarán por una etapa llamada de formación antes de que puedan ser sometidas a un entorno de mercado (Jacobsson y Bergek, 2004). En esta fase, los actores se incorporan en el siT 
para iniciar con la creación de redes y el diseño de las instituciones que hacen que la tecnología se acople a las estructuras que la rodean. En el periodo de formación se perfilan los diversos componentes necesarios para constituir el sIT, por ejemplo, la acumulación de conocimientos y configuraciones institucionales (Edsand, 2019).

El proceso de creación del sit se conceptualiza a partir de las funciones que representan actividades esenciales para el desarrollo del sistema que promuevan el avance tecnológico. Las funciones fomentan la acumulación del sit y acelera el crecimiento del sistema a través de mecanismos de retroalimentación positiva de las funciones. Este proceso fue denominado causalidad acumulativa (Suurs, 2009; Suurs y Hekkert, 2009a; Suurs et al., 2009b) y se refiere a que las funciones del sistema interactúan y se refuerzan entre sí con el tiempo. En la literatura de sIT dicho proceso se conoce como motores de innovación y se analiza con respecto a la estructura y las funciones del sistema. Ambos elementos deben considerarse como dos aspectos diferentes de la cuestión analizada.

La estructura del sit consta de tres componentes: actores, instituciones y tecnología. i) Los actores involucran a las organizaciones que contribuyen al surgimiento de la tecnología emergente, entre los que se encuentran: desarrolladores, adoptantes, financiadores, etcétera. ii) Las instituciones se definen con base en North (1990) y constituyen las leyes, los reglamentos y las normas que regulan el contexto. iii) Las tecnologías son los artefactos y las infraestructuras tecnológicas en las que se integran (Suurs et al., 2009b). Los procesos que se dan dentro del sistema se representan a través de siete funciones que se deben cumplir para que se genere desarrollo y difusión tecnológica.

\section{Funciones del SIT y motores de innovación sustentable}

Las funciones representan actividades necesarias para la acumulación del sIT; la tabla 1 ofrece una visión general de sus implicaciones y eventos con los que se pueden relacionar para su identificación. El trabajo utiliza la propuesta de análisis funcional de Hekkert et al. (2007) para el análisis del caso empírico.

En etapas formativas de los SIT, las funciones del sistema presentan dinámicas particulares definidas por el grado de desarrollo de sus componentes. Es decir, su desempeńo se condiciona a la madurez, por ejemplo, si los actores están definidos y el desarrollo tecnológico está presente, necesariamente tiene que haber un ajuste institucional que acompańe dichas innovaciones para que en el largo plazo se promueva la difusión tecnológica. 
Diana Rivera Delgado, Fernando J. Díaz López y Graciela Carrillo González

Tabla 1. Funciones del SIT y sus eventos asociados

\begin{tabular}{|c|c|c|}
\hline \multicolumn{2}{|c|}{ Función } & Eventos \\
\hline$[\mathrm{Fl}]$ & Actividades de emprendimiento & $\begin{array}{l}\text { Proyectos con fines comerciales, empresas nuevas, empresas establecidas, combinación } \\
\text { de empresas del sector público y privado. }\end{array}$ \\
\hline$[\mathrm{F} 2]$ & Desarrollo de conocimiento & $\begin{array}{l}\text { Publicaciones, patentes, proyectos de investigación, proyectos nacionales, investigación } \\
\text { de conocimiento básico, investigación para el desarrollo tecnológico. }\end{array}$ \\
\hline [F3] & Difusión de conocimiento & $\begin{array}{l}\text { Talleres, conferencias, exposiciones, vinculación internacional, colaboraciones } \\
\text { tecnológicas, relaciones industria-academia. }\end{array}$ \\
\hline [F4] & Orientación de la investigación & $\begin{array}{l}\text { Desarrollo de una visión y objetivos por parte del gobierno, desarrollo de áreas } \\
\text { prioritarias, incentivos, apoyos, normas complementarias. }\end{array}$ \\
\hline$[\mathrm{F} 5]$ & Formación de mercado & $\begin{array}{l}\text { Ventajas competitivas de mercado por medio de incentivos y apoyos, compra } \\
\text { gubernamental, alineación de políticas económicas y comerciales nacionales. }\end{array}$ \\
\hline [F6] & Movilización de recursos & $\begin{array}{l}\text { Recurso humano especializado, inversión en I+D pública y privada, incubadoras, parques } \\
\text { tecnológicos, centros de investigación especializados. }\end{array}$ \\
\hline$[\mathrm{F} 7]$ & Creación de la legitimidad & $\begin{array}{l}\text { Desarrollo de instituciones y reglamentes especiales, normas, derechos de propiedad } \\
\text { intelectual. }\end{array}$ \\
\hline
\end{tabular}

Fuente: Hekkert et al. (2007).

Los motores se definen con base en las funciones dominantes que participan en el sistema y considerando el nivel de desarrollo de los elementos. Sirven para explicar cómo se da la formación de los sistemas cuando se cuenta con diversas características. Resulta relevante mencionar que las funciones del sistema no interactúan directamente sino que lo hacen a través de los motores de innovación, es decir, los actores del sistema actúan de acuerdo con las condiciones institucionales y tecnológicas existentes, creando y apoyando eventos que contribuyen al desarrollo del sit en general (véase tabla 2).

Los motores están relacionados con factores estructurales, algunos tienden a construirse a partir de la existencia anterior de otros. Dicha característica hace que los SIT se desarrollen en configuraciones estructurales más completas y complejas, que a su vez son propensas a estimular el desarrollo de motores más avanzados y con ello llegar a la etapa de crecimiento. Los mecanismos de cada motor no son lineales, e implican una variedad de funciones conectadas a través de relaciones de distinta intensidad que generan retroalimentación. Los SIT no necesariamente experimentan todas estas etapas, ya que pueden colapsar y no desarrollarse más. Comprender las funciones del sistema y los motores que explican su formación es de gran relevancia para analizar las características del sit de udeg en México. 
Tabla 2. Motores de innovación sustentable

Motor

Caracteristicas generales
Empuje de la Ciencia y

la Tecnología (ECT)

Empresarial

Construcción del sistema

Mercado
Su dinámica se caracteriza por un fuerte cumplimiento de las funciones de desarrollo de conocimiento [F2], difusión de conocimiento [F3], orientación de la investigación [F4] y movilización de recursos [F6]. El motor comienza con la orientación [F4], se apoya en la movilización de los recursos [F6], lo que resulta en desarrollo y difusión de conocimientos necesarios para la tecnología emergente [F2, F3], de ahí termina el ciclo y regresa a [F4] donde comenzó en el primer lugar. Un segundo ciclo va de $[\mathrm{F} 4]$ a $[\mathrm{F} 6]$ a $[\mathrm{Fl}]$ a $[\mathrm{F} 4]$, pero esta nueva etapa de retroalimentación suele ser débil. La Formación de Mercado [F5] está ausente, ya que en esta parte la actividad empresarial no tiene gran participación aunado a que el motor puede continuar su dinámica incluso sin el apoyo de la industria. Surge una visión compartida que proporciona una dirección al campo tecnológico. Además el número de científicos y empresas aumenta y sus relaciones se hacen más fuerte, cobijados bajo el establecimiento de instituciones oficiales de apoyo.

Se identifica por un fuerte cumplimiento de las funciones de desarrollo de conocimiento [F2], difusión de conocimiento [F3], orientación de la investigación [F4] y movilización de recursos [F6]. Lo que diferencia al Motor Empresarial del ECT es la fuerte presencia de la función de creación de legitimidad [F7] y especialmente las actividades empresariales [Fl]. La secuencia de eventos que caracteriza a este motor comienza con las empresas, los servicios públicos y/o los gobiernos locales que entran en el SIT e inician proyectos innovadores [Fl], generalmente experimentos de adopción o proyectos de demostración, porque ven oportunidades de negocio [F4], con estas condiciones los actores ejercen presión al gobierno [F7], si los resultados son positivos se da la movilización de recursos [F6]. Los resultados retroalimentan y determinan la dinámica ya que proporcionan la caracterización del escenario para que otros actores se integren al sistema.

Implica la participación de todas las funciones propuestas en la literatura. La adición importante es la formación de mercado [F5], que apenas estaba presente en el motor ECT. La secuencia de eventos asociada a este motor comienza con las empresas y otros actores que se aventuran en proyectos innovadores, típicamente demostraciones, a veces con resultados exitosos [F1, F4]. Como parte de estos emprendimientos, estos actores se organizan en plataformas para compartir conocimientos, pero también para comunicar y coordinar el desarrollo tecnológico posterior [F2, F3, F4]. En el marco de estas plataformas, también presionan para obtener recursos [F6, F7]. Este motor da lugar a que el gobierno nacional y las grandes empresas se conviertan en una parte integral del sistema. En el sector de la energía, dichas empresas suelen controlar infraestructuras importantes. Los actores del sistema forman fuertes redes que inducen mayor desarrollo, además de necesitar un respaldo institucional específico que acompañe la tecnología.

Se caracteriza por una fuerte contribución a las Actividades Empresariales [Fl], Desarrollo de Conocimientos [F2], Difusión de Conocimientos [F3], Orientación de la Búsqueda [F4], Movilización de Recursos [F6] y Formación de Mercado [F5]. Todas las funciones del sistema están fuertemente cumplidas excepto el apoyo de las Coaliciones de Defensa [F7], que no es tan importante para este motor. La razón principal es que la Formación del Mercado [F5] ya no es un asunto de política; se ha creado un ambiente de mercado como resultado de regulaciones formales. En su lugar, la Formación de Mercados [F5] se adopta como parte de las actividades empresariales regulares, es decir, estrategias de marketing y promoción que están directamente vinculadas a las Actividades Empresariales [Fl].

Fuente: elaboración propia con base en Suurs y Hekkert (2012). 


\section{USOS DIRECTOS DE ENERGÍA GEOTÉRMICA EN MÉXICO (UDEG)}

En México, el artículo 2 del Reglamento de la Ley de Energía Geotérmica (DOF, 2014), define como usos directos a aquellos en los que se puede aprovechar la energía geotérmica que sean diferentes a la generación de electricidad como: calefacción urbana o de invernaderos, secado de productos agrícolas o industriales, entre otros. México dispone de un gran potencial de recursos geotérmicos que en su mayoría se utilizan para la generación de electricidad.

A nivel institucional, México dinamizó el desarrollo de energías renovables (ER) en el periodo 2008-2019. En esta etapa se dieron cambios en el marco institucional energético para favorecer el desarrollo y la difusión de las ER y con ello aumentar su participación en la matriz energética nacional. Lo anterior generó la promulgación de leyes, acuerdos, tratados y estrategias de financiamiento que establecen el direccionamiento de la transición energética y coadyuvan a su desarrollo a nivel nacional.

En 2013 se creó el Fondo de Sustentabilidad Energética (FSE) cuyo propósito fundamental era impulsar a la ciencia, tecnología e innovación en materia de sustentabilidad energética a través de distintas acciones y priorizando la vinculación de diversos actores del sistema. En ese mismo año se publicó la Ley para el Aprovechamiento de las Energías Renovables y el Financiamiento de la Transición Energética (LAERFte) que estableció una meta de 35\% de generación eléctrica basada en ER para el 2024. Para lograrlo, se propuso el desarrollo de conocimientos y tecnologías para las ER.

El fSE sirvió para crear los Centros Mexicanos de Innovación en Energía Renovable (CEMIE), arrancando con el CEMIE-Geo que formó una alianza academia-industria con el apoyo de la Secretaría de Energía (sENer) y el Consejo Nacional de Ciencia y Tecnología (CONACYT) y tuvo como objetivo promover y acelerar el uso y desarrollo de la energía geotérmica (Jones y Kretzschmar, 2017). Las instituciones académicas que conformaron el consorcio fueron: i) Centro de Investigación Científica y de Educación Superior de Ensenada, CICESE, ii) Instituto Nacional de Electricidad y Energías Limpias, INEel, iii) Universidad de Guadalajara, UDG, iv) Universidad Michoacana de San Nicolás de Hidalgo, umsnh, v) Centro de Geociencias, unam, vi) Instituto de Energías Renovables, UnAm, vii) Instituto de Geofísica, UnAM, viii) Instituto de Geología, unam, $i x$ ) Instituto de Ingeniería, unam, $x$ ) Universidad Politécnica de Baja California, upbc, xi) Centro de Tecnología Avanzada A.C., CIATEQ y empresas privadas. 
Tabla 3. Proyectos de usos directos calor geotérmico de CEMIE-Geo, 2014-2018

\begin{tabular}{|c|c|c|c|c|c|}
\hline Aplicaciones & Tecnología & Ubicación & Actores & $\begin{array}{l}\text { Etapa de } \\
\text { desarrollo }\end{array}$ & $\begin{array}{l}\text { Tipo de } \\
\text { desarrollo }\end{array}$ \\
\hline Climatización de edificio & $B C G$ & Morelos & INEEL & $\begin{array}{l}\text { Estudio de mercado } \\
\text { y anteproyecto }\end{array}$ & AT \\
\hline Climatización de laboratorios & $B C G$ & Baja California & INEEL y UPBC & En construcción & AT \\
\hline $\begin{array}{l}\text { Acondicionamiento de } \\
\text { espacios, climatización de } \\
\text { escuela y centro de salud }\end{array}$ & $B C G$ & Puebla & INEL y CFE & Demostración & AT \\
\hline $\begin{array}{l}\text { Acondicionamiento de espacios } \\
\text { (hotelería) }\end{array}$ & $B C G$ & Guerrero & Empresa privada & Comercial & AT \\
\hline Sistema de climatización & $B C G$ & Ciudad de México & II, UNAM & Demostración & DT \\
\hline Climatización de invernadero & $B C G$ & Baja California & INEEL y UPBC & Demostración & AT \\
\hline Climatización de edificios & $B C G$ & Michoacán & UMSNH & Demostración & DT \\
\hline Deshidratador de alimentos & $\mathrm{DA}$ & Nayarit & Grupo iiDEA & Operación & DT \\
\hline Climatización de oficinas & $B C G$ & Michoacán & CFE & Operación & AT \\
\hline Invernadero de hortalizas & $B C G$ & Jalisco & CEO Hortalizas & Comercial & AT \\
\hline Desaladora de agua de mar & DS & Baja California Sur & Grupo ïDEA & Demostración & DT \\
\hline Deshidratador de alimentos & $\mathrm{DA}$ & Michoacán & UMSNH & Piloto & DT \\
\hline
\end{tabular}

Notas: BCG: bomba de calor geotérmico; DA: deshidratador de alimentos; DS: desaladora de agua de mar; AT: apropiación tecnológica; DT: desarrollo tecnológico.

Fuente: elaboración propia a partir de entrevistas.

El CEMIE-Geo desarrolló un Sistema de Laboratorios Especializados (SLE), en los que se profundizó el conocimiento del recurso a través de varias líneas de investigación, una de ellas fue UDEG, que durante el periodo 2014-2018 realizó proyectos que tuvieron como propósito general el desarrollo de capacidades tecnológicas y la generación de conocimiento asociado a las tecnologías para diversas aplicaciones del recurso (véase tabla 3).

La creación del centro y el financiamiento para el desarrollo de estos proyectos de UDEG, muestra la orientación de la investigación para el desarrollo de tecnologías relacionadas con la industria geotérmica. Los actores del sit de UDEG promovieron el desarrollo de conocimiento, ya que durante el periodo 2008-2019 se generaron publicaciones científicas y de divulgación, así como tesis de licenciatura y maestría sobre tecnologías y aplicaciones de usos directos. En 2011 se hicieron evaluaciones económicas y tecnológicas al recurso, en 
vinculación con organismos internacionales como el Banco Interamericano de Desarrollo. A partir de 2014 se ofertaron cursos cortos en diferentes disciplinas geotérmicas y también incentivaron la vinculación con expertos a través de diversos proyectos y por medio de la realización de congresos, simposios y talleres nacionales e internacionales en materia de UDEG para la difusión del conocimiento. En términos de generación de capital humano especializado se incluyeron planes curriculares en programas de posgrado en las instituciones académicas que conformaron el centro.

\section{PANORAMA METODOLÓGICO: ANÁLISIS HISTÓRICO DE EVENTOS Y MODELADO TEMÁTICO}

El objetivo del presente trabajo es identificar las funciones existentes en el SIT de UDEG para determinar el grado de desarrollo del sistema y determinar a qué tipo de motor de innovación corresponde. En primer lugar se trazó un mapa de las principales actividades y procesos históricos relacionados con el sit de Udeg. Para ello se adoptó el análisis histórico de eventos (AHE), propuesto por Van De Ven y Poole (2005). Esta metodología permitió operar y medir las funciones del sistema relacionándolas con eventos, que se definen en términos de los temas discutidos en los documentos analizados. Los eventos ayudaron a identificar categorías representativas de las funciones presentes en el sistema. La interacción entre las funciones del sistema se midió mediante el seguimiento de las secuencias de eventos que se repitieron a lo largo del periodo 2008-2019.

Para medir lo anterior se aplicó la técnica de clasificación con métodos no supervisados de modelado temático (MT), que implica el procesamiento automatizado de un cuerpo de textos para determinar los temas subyacentes del conjunto de documentos (Blei et al., 2003; Mohr y Bogdanov, 2013). Los algoritmos del MT son métodos estadísticos que analizan: i) las palabras de los textos originales para descubrir los temas latentes que son transversales; ii) cómo se conectan esos temas entre sí, y iii) cómo cambian en el tiempo (Blei et al., 2012).

El Mт busca patrones recurrentes en el contenido de los textos y lo hace con base en la frecuencia de aparición de palabras, dicha técnica facilita la identificación de los temas latentes que estructuran un corpus de documentos (Blei et al., 2012). Existen diversos algoritmos para hacer MT, entre los que se encuentran: Latent Dirichlet Allocation (LDA), Pachinko Allocation, Hierarchical LDA, entre otros (Nowlin, 2016). 


\section{Modelos LDA}

Son una herramienta útil para el análisis de las funciones del sit y se definen como un modelo estadístico del lenguaje, en el que cada documento dentro de un set o corpus es visto como una bolsa de palabras producidas de acuerdo a una mezcla de temas que el autor del texto pretendía discutir. Cada tema es una distribución sobre todas las palabras observadas en el corpus, de tal manera que los términos que están fuertemente asociados con los temas dominantes del documento tienen una mayor probabilidad de ser seleccionados. Para correr el modelo LDA fue necesario desarrollar un algoritmo en el lenguaje y ambiente para cómputo estadístico y gráfico RStudio.

Entre los resultados que arroja el modelo LDA se encuentra un conjunto de distribuciones temáticas por palabras que asocian una probabilidad con cada par de palabras temáticas y un conjunto similar de distribuciones de documentos por temas, que describen la probabilidad de elegir un tópico particular para cada documento del corpus. La estructura obtenida es latente, lo que implica que las distribuciones temáticas por palabras no están asociadas con una etiqueta explicita de un tema, sino con un conjunto de probabilidades de palabras que cuando se ordenan por probabilidad decreciente a menudo se relacionan estrechamente y, por lo tanto, se conciben como "temas". La figura 2 presenta de forma sintética los pasos del modelo LDA.

Figura 2. Diagrama esquemático que muestra la secuencia usada para el desarrollo del modelo LDA

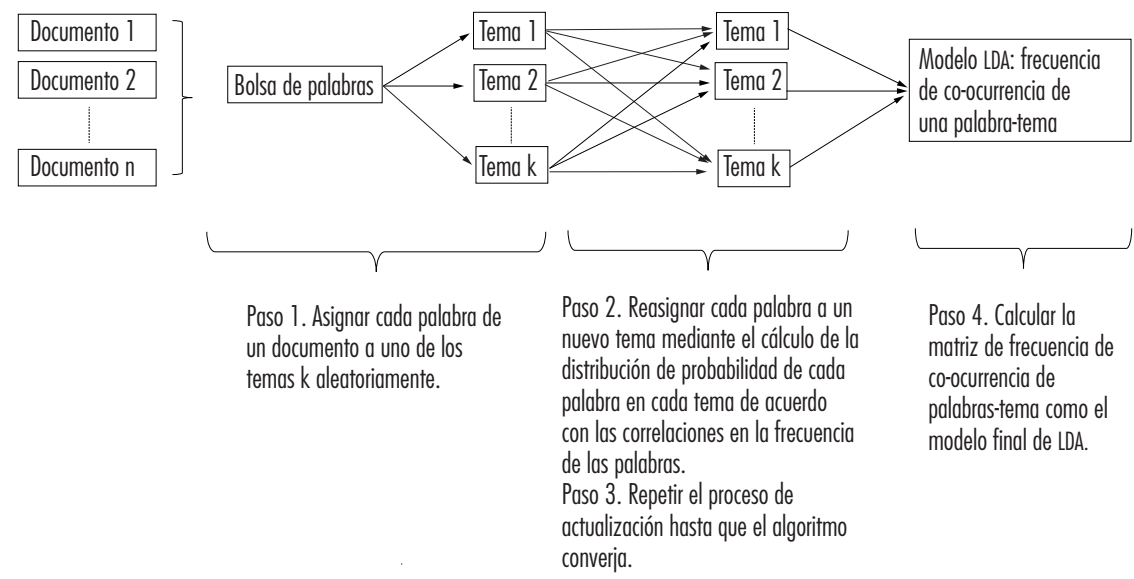

Fuente: elaboración propia, diseñada de la metodología sugerida por Ding et al. (2018). 


\section{Datos del SIT de UDEG en México}

Se analizaron 125 documentos relacionados con el SIT de UDEG del periodo 2008-2020, 60 de ellos comprenden leyes, normas y estrategias desarrolladas por el gobierno para acelerar la transición energética y el desarrollo de las ER. El resto de los documentos (65) abarca la información relacionada con el desarrollo y la difusión de conocimientos y tecnologías para UDEG, generada en el periodo estudiado.

Para estudiar al componente instituciones se examinaron leyes, normas y estrategias propuestas por el gobierno para las ER. Por otra parte, para analizar al componente actores se recabó información relacionada con el desarrollo y la difusión de conocimientos y tecnologías de UDEG, en el mismo periodo. En la tabla 4 se observa su clasificación, con ellos se elaboraron dos modelos que reúnen la mayor cantidad de eventos en el periodo estudiado. Los datos de diferentes fuentes se triangularon para asegurar la exactitud (Yin, 2009).

Los resultados obtenidos de los modelos se codificaron en las funciones utilizando los eventos propuestos en la tabla 1. Para ello, se razonó que el hecho de que un suceso se clasificara en una función determinada representaba una prueba de que esa función existía y que, una vez establecida una función, su impacto persistía a lo largo del tiempo. Por lo tanto, se considera que las funciones son aditivas y se acumulan a lo largo del tiempo, lo que aumenta la capacidad de los actores y la capacidad del sistema en su conjunto para cumplir las funciones.

Se calculó el número ideal de temas (k) -de acuerdo con Blei et al. (2003)-; determinar el número de $\mathrm{k}$ es uno de los mayores desafíos cuando se utilizan los modelados de temas, ya que en los enfoques no supervisados, el investigador es responsable de que las categorías derivadas del modelo sean justificables con base en lo que se quiere demostrar. Para que un tema sea representativo debe de cumplir con la validez semántica, que implica que cada tema tiene un significado claro y coherente que puede detectarse por la asociación de los términos en ese tema (Grimmer y Stewart, 2013). El número ideal de k debe seleccionarse en función de la pregunta de investigación y estar guiado por la teoría. Después de correr varios modelos con $\mathrm{k}>4$, donde los resultados no eran representativos ni aportaban a la explicación, se determinó $\mathrm{k}=4$, ya que era representativo para el análisis. 
Tabla 4. Documentos analizados

\begin{tabular}{lcc}
\hline Tipo de documento & Núm. de documentos & Modelo \\
\hline $\begin{array}{l}\text { Programas, estrategias, lineamientos y marcos normativos en materia } \\
\text { de energías renovables. }\end{array}$ & 48 & LDA-Instituciones \\
Documentos oficiales relacionados las tecnologías para UDEG. & 12 & \\
Artículos de divulgación y talleres/conferencias. & 29 & LDA-Actores \\
Artículos científicos. & 11 & 16 \\
Evaluaciones para tecnologías de UDEG. & & \\
Entrevistas con actores clave: líderes de proyectos de UDEG de CEMIE- & 9 & \\
GEO, consultores externos de la Comisión Federal de Electricidad y del \\
Instituto Mexicano del Petróleo.
\end{tabular}

Fuente: elaboración propia.

\section{ANÁLISIS DE RESULTADOS}

Se realizaron dos modelos LDA, en el primero se analizó a las instituciones que acompañaron el desarrollo del sit. El segundo examinó los documentos relacionados con el desempeño de los agentes del sistema, aquello relacionado con generación y difusión de conocimiento, ambos para el periodo 20082019. El objetivo era conocer y caracterizar a nivel estructural el desempeño de las funciones y, con ello, comprender el motor de innovación sustentable que predomina en el SIT de UDEG.

En las figuras 3 y 4 se presentan los resultados del TM de los modelos desarrollados. En la parte izquierda se muestran los temas en el plano bidimensional y se representan como círculos, el tamaño de ellos indica la relevancia de cada uno. En el panel derecho se muestra un gráfico de barras horizontales que representan los términos individuales más útiles para interpretar cada tema. 

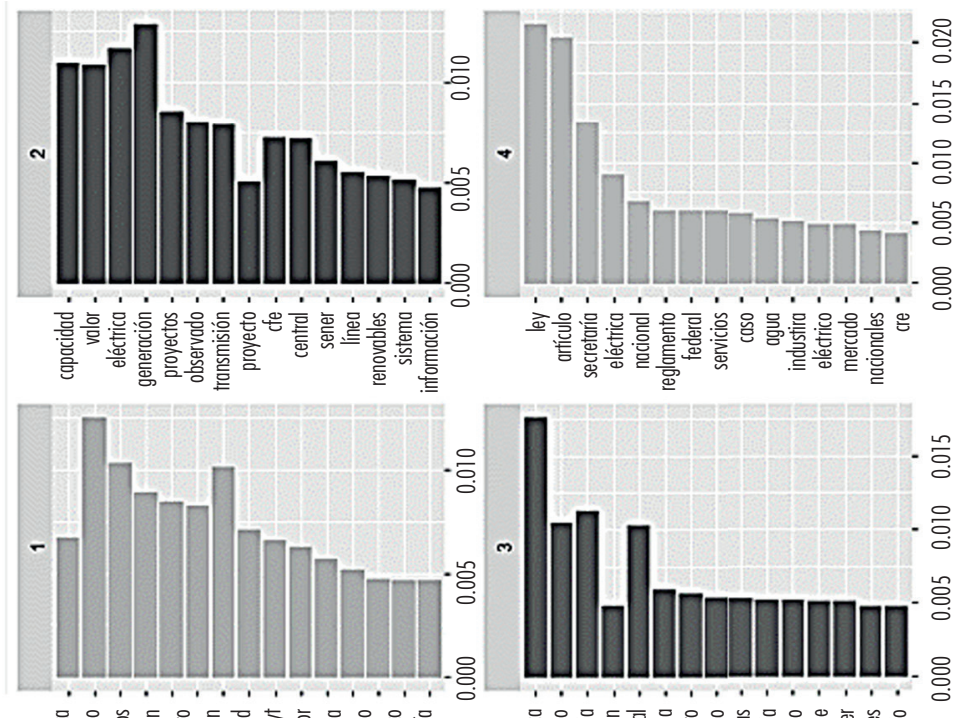

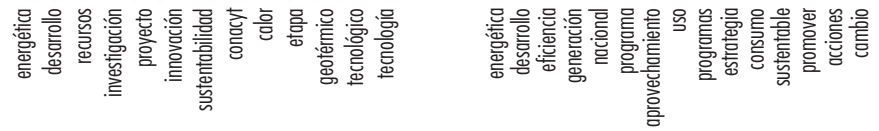

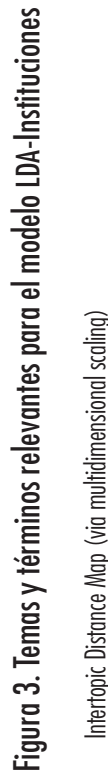

แมગ
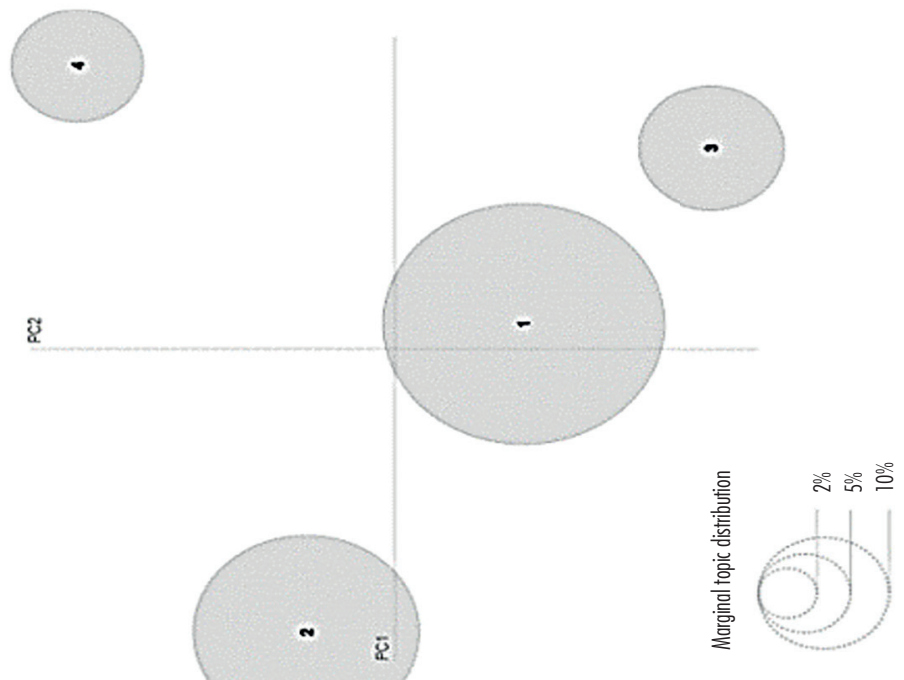

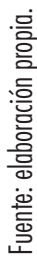



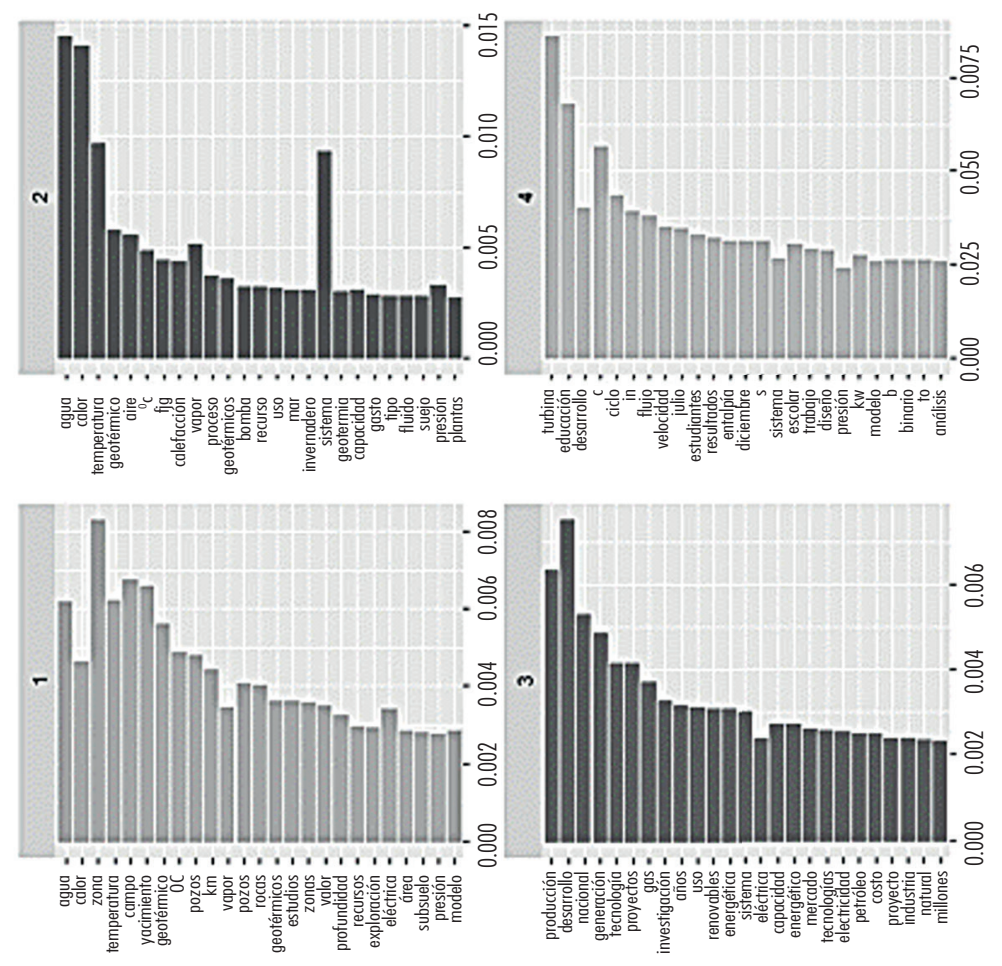

แมว
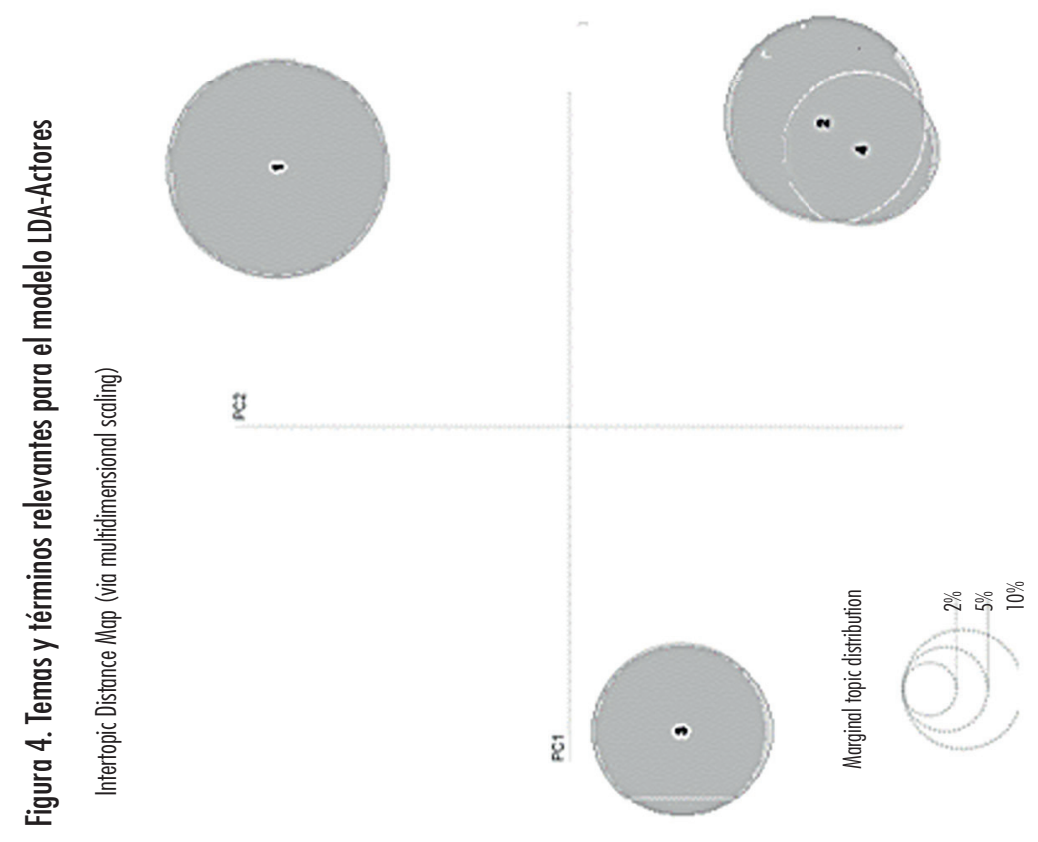

흥
응
등
응
흥
힘
홍 


\section{LDA-Instituciones}

En el análisis institucional se puede observar con claridad que los temas no se sobreponen, ya que responden a distintas funciones del sistema. Esto es así, al ir de lo plural a lo específico, en el sentido de que en este análisis el corpus se compone de las estrategias nacionales para el desarrollo de energías renovables y promoción de tecnologías limpias, expresado en la de Ley de Cambio Climático, Ley de Aguas Nacionales, Ley de la Industria Eléctrica, Ley de Energía Geotérmica, etcétera, y también de instrumentos institucionales específicos de UDEG (como mapas de ruta y diagnósticos tecnológicos y de capacidades). A nivel institucional se promovió la Ley de Transición Energética (LTE) que establece la Estrategia de Transición para Promover el Uso de Tecnologías y Combustibles más Limpios, dicho instrumento fue el rector de la política nacional de energías limpias. Lo relevante de analizar los documentos mencionados, fue conocer cómo se planteó la participación de la ciencia y la tecnología para garantizar el incremento de la participación de las ER, puesto que gran parte de la estrategia nacional se basa en la mejora de la eficiencia energética a través del conocimiento y las mejoras tecnológicas.

En la figura 3 se puede observar que el tema 1 es el más representativo y discutido a lo largo del set de documentos y constituye la función de "orientación de la investigación”. Esto es así, ya que el gobierno delimitó los parámetros con los que buscó impulsar la TE, para ello definió metas a largo plazo e instrumentos que le ayudarían a cumplirlas. El tema dos es el que sigue en importancia y caracteriza la función de "creación de legitimidad", pues fue necesario la elaboración de normas que validaran los esfuerzos. El tema 3 representó la función de generación y difusión de conocimiento, como se mencionó anteriormente, las estrategias que se plantearon se basaron en promover la innovación y el desarrollo de conocimiento para las ER. El tema 4 constituyó la función de desarrollo de conocimiento para ER y el sector energético de manera general. Del lado derecho de la imagen se muestran los top 15 términos de cada tema.

En la tabla 5 se resumen los términos más representativos de cada tema y la función que tuvieron con base en la validez semántica que presentó cada uno de ellos. Específicamente para UDEG, la legislación promovió la identificación y unión de los actores relevantes del SIT, por medio del CEMIE-Geo, ya que a partir de la creación del consorcio se generó una red de interacción entre los participantes y se promovió la vinculación con la industria. Esto fue importante, ya que antes de que existiera el centro, los esfuerzos en materia de desarrollo de UDEG en México estaban desarticulados. 
Tabla 5. Relación entre tópicos y funciones del SIT derivadas del LDA-Instituciones

\begin{tabular}{lll}
\hline Tópico & \multicolumn{1}{c}{ Términos } & Función que representan \\
\hline 1 & $\begin{array}{l}\text { Energética, eficiencia, desarrollo, nacional, programa, aprovechamiento, uso, } \\
\text { programa, estrategia, consumo, sustentable, proyectos, promover, generación, } \\
\text { acciones, cambio, tecnologías, transición. }\end{array}$ & Orientación de la investigación \\
2 & $\begin{array}{l}\text { Ley, artículo, secretaría, nacional, reglamento, federal, servicios, agua, industria, } \\
\text { eléctrico, mercado, nacionales, CRE, uso, derechos. }\end{array}$ & Creación de legitimidad \\
3 & $\begin{array}{l}\text { Desarrollo, recursos, proyecto, investigación, fondo, innovación, energética, } \\
\text { sustentabilidad, CoNACYT, calor, geotérmico, tecnológico, informe, nacional, } \\
\text { sectorial, CEMIE, usos, directos. }\end{array}$ & $\begin{array}{l}\text { Desarrollo y difusión de } \\
\text { conocimiento }\end{array}$ \\
\hline & $\begin{array}{l}\text { Generación, capacidad, valor, proyectos, transmisión, sistema, SENER, sistema, } \\
\text { proyecto, información, CFE, recursos, millones, distribución, potencia, gas, } \\
\text { nacional. }\end{array}$ & $\begin{array}{l}\text { Desarrollo de conocimiento, para la } \\
\text { infraestructura del sector energético }\end{array}$ \\
\hline
\end{tabular}

Fuente: elaboración propia.

\section{LDA-Actores}

Partiendo del hecho que institucionalmente se buscó el desarrollo del conocimiento y de tecnologías para UDEG, en la figura 4 se puede observar que el tema 1 representa la función de "orientación de la investigación" en términos del desarrollo, creación de conocimientos e innovación. Los proyectos se alinearon en la búsqueda de la eficiencia energética y un sector energético renovado a través de este tipo de recurso. El tema 2 representa la "creación y difusión del conocimiento", en este se habla de innovación, aplicaciones y tecnologías para explotar los UDEG. El tema 3 representó la movilización de los recursos, tanto financieros como el capital humano especializado, la educación y el desarrollo de proyectos fueron relevantes. El tema 4 constituyó la función de desarrollo de conocimiento específico para el recurso. Términos como calor, temperatura, vapor y agua fueron comunes entre el tema 2 y 4 , pues ambos temas discuten el desarrollo de conocimiento, uno enfocado en el recurso (geotermia y UDEG) y el otro en las tecnologías o aplicaciones del recurso (bombas de calor, invernaderos, etcétera). Esto es importante para el desarrollo del SIT, pues al estar en etapa formativa ambas funciones son de principal importancia para construir las bases del sistema.

En la tabla 6 se resumen los términos más representativos de cada tema y la función que representaron con base en la validez semántica que presentó cada uno de ellos. Se puede observar que la orientación de la investigación llevó a los actores del sistema tanto a investigar sobre las tecnologías para la explota- 
ción de UDEG, como en las aplicaciones comerciales que se podrían hacer. Esto es relevante, ya que los UDEG no compiten directamente con la generación eléctrica con la energía solar o eólica, sino tienen la ventaja de poder ser aplicados en sectores económicos diversos, por lo que el tema 2 da claridad hacia dónde se dirigió el esfuerzo de los actores en la creación del conocimiento.

En la tabla 7 se observan los hallazgos de esta investigación, al identificar las funciones relacionadas con los eventos resultantes de ambos modelos LDA. Resalta que para ambos componentes la orientación de la investigación fue el tema de mayor importancia, tal como se aprecia en las figuras 3 y 4.

\section{Tabla 6. Relación entre tópicos y funciones del SIT derivadas del LDA-Actores}

\begin{tabular}{lll}
\hline Tópico & \multicolumn{1}{c}{ Términos } & \multicolumn{1}{c}{ Función que representan } \\
\hline 1 & $\begin{array}{l}\text { Desarrollo, producción, nacional, generación, gas, tecnología, renovables, } \\
\text { investigación, energética, mercado, industria, emisiones, innovación, natural, costos. }\end{array}$ & Orientación de la investigación \\
2 & $\begin{array}{l}\text { Agua, calor, temperatura, sistema, aire, calefacción, geotérmico, vapor, invernadero, } \\
\text { bombas, desalación, mar, proceso, secado, humedad. }\end{array}$ & $\begin{array}{l}\text { Desarrollo y difusión de } \\
\text { conocimiento tecnológico para las } \\
\text { aplicaciones del recurso }\end{array}$ \\
3 & $\begin{array}{l}\text { Educación, desarrollo, flujo, estudiantes, resultados, análisis, estatal, sistema, } \\
\text { escolar, trabajo, ciclo, escuela, superior, diseño, modelo. }\end{array}$ & $\begin{array}{l}\text { Movilización de recurso, en este } \\
\text { caso se hace referencia al capital }\end{array}$ \\
& $\begin{array}{l}\text { Zonamano especializado } \\
\text { estudios, profundidad, recursos, exploración, subsuelo, presión. }\end{array}$ & $\begin{array}{l}\text { Desarrollo de conocimiento } \\
\text { espećfico para el recurso }\end{array}$ \\
\hline
\end{tabular}

Fuente: elaboración propia.

\section{Tabla 7. Funciones presentes en el SIT de UDEG por componente}

\begin{tabular}{lll}
\hline Tema & \multicolumn{1}{c}{ Funciones identificadas en el componente Actores } & \multicolumn{1}{c}{$\begin{array}{c}\text { Funciones identificadas en el } \\
\text { componente Instituciones }\end{array}$} \\
\hline 1 & Orientación de la investigación [F4]. & Orientación de la investigación [F4]. \\
2 & $\begin{array}{l}\text { Desarrollo y difusión de conocimiento tecnológico para las aplicaciones } \\
\text { del recurso [F2 y F3]. }\end{array}$ & Creación de legitimidad [F7]. \\
3 & Movilización de recurso, en este caso se hace referencia al capital & Desarrollo y difusión de conocimiento \\
humano especializado [F6]. & tecnológico [F2 y F3]. \\
4 & Desarrollo de conocimiento específico para el recurso [F2]. & Desarrollo de conocimiento, para la \\
& & infraestructura del sector energético [F2]. \\
\hline
\end{tabular}

Fuente: elaboración propia. 
Complementando lo anterior, también se destaca que en el caso específico del sit de udeg en México, el desarrollo de conocimiento se discute en dos vertientes para ambos componentes: para los actores se desarrolla y difunde conocimiento en términos de las aplicaciones/tecnología, pero también es importante abonar al conocimiento de las características del recurso geotérmico. Algunas de las otras líneas de investigación del CEMIE-Geo profundizan con mayor intensidad en el conocimiento del recurso. Por otra parte en el componente institucional, también se dan dos tipos de desarrollo de conocimiento y se hace en términos de las tecnologías, aunque más enfocadas en temas de sustentabilidad y de infraestructura del sector energético.

Con base en lo anterior y considerando las funciones presentes en la discusión de cada componente se puede decir que el sit de UDEG en México comienza con la orientación de la investigación [F4], ya que el gobierno propone el desarrollo de CEMIE-Geo y congrega a distintos actores para que lo conformen. Para lograrlo, dota de recursos financieros [F6] que permiten el desarrollo de proyectos de investigación y tecnologías a nivel prototipo, además de apoyar con financiamiento para recurso humano especializado. Esto a nivel nacional se centra en el desarrollo de conocimientos, tanto tecnológicos como del recurso, la difusión se hace con menor escala y es que los eventos y las vinculaciones nacionales y extranjeras se han promovido, pero no priorizado, por lo que los recursos en este caso determinan la aparición y el reforzamiento de [F2 y F3], ahí termina el ciclo y regresa a F4 donde el ciclo comenzó en el primer lugar.

La Formación de Mercado [F5] está ausente, ya que en esta parte la actividad empresarial no tiene gran participación, pues este sIT se ha concentrado y desarrollado en el sector académico y aquellas empresas que participan son spin off de la misma academia, a pesar de lo anterior se enfoca en el desarrollo de conocimiento de aplicaciones con fines comerciales. Si bien a nivel institucional es importante la creación de legitimidad [F7], esto es lógico, ya que el análisis del componente se basó en el marco normativo propuesto para incentivar el desarrollo de las energías renovables. Los impactos estructurales en sit del motor se relacionan con el surgimiento de una visión compartida que proporciona una dirección al campo tecnológico. Durante el periodo 20142019 el número de científicos y empresas (spin off) aumentaron y debido a que el CEMIE-Geo los reunió en un consorcio específico de geotermia, las relaciones de los científicos se estrecharon bajo el establecimiento de instituciones oficiales de apoyo, se puede decir entonces que el motor de innovación en el que se encuentra el sit de Udeg en México es el de Empuje de la Ciencia y la Tecnología y se encuentra en una etapa de formación como sistema, pero también buscando el desarrollo de sus componentes. 


\section{CONCLUSIONES}

La evidencia muestra que el sIT de UDEG se encuentra en una etapa de formación, en la que sus componentes no se encuentran debidamente desarrollados para el cumplimiento de las siete funciones propuestas por la literatura. A pesar de ello, la formación del sit responde a cuatro funciones que fueron direccionadas por la orientación de la investigación [F4] que dio entrada a la movilización de recursos [F6] y la combinación de ambas funciones generó el desarrollo y la difusión de conocimiento [F2y F3], esto representó la primera fase del sistema. También se evidencia que la creación de legitimidad [F7] jugó un papel relevante al ser el acompañante principal de la formación del sistema con una serie de políticas e instrumentos que abonaron a su desarrollo, esto es relevante, ya que se diferenció de lo propuesto teóricamente.

Con base en lo anterior, se puede decir que el motor que define la formación del sit de UDEG en México se relaciona con el de Eст y dicho mecanismo puede ser altamente influyente en los países emergentes, ya que genera nichos para el desarrollo de tecnologías que están libres de fallos de mercado y de sistema, tal como en el caso nacional. Sin embargo, es imperativo considerar que los ambientes protegidos tienen un límite de crecimiento. Este estudio demuestra que el apoyo gubernamental a través de la orientación de instrumentos institucionales hacia una visión de ER, así como el desarrollo de conocimientos y tecnologías llevó a la formación de una parte del sit de UDEG, e identificó sus actores principales.

Para lograr los objetivos de la transición energética es fundamental que el Estado genere políticas públicas orientadas a su rápida y adecuada puesta en marcha. Los desarrollos generados por CEMIE-Geo representan alternativas energéticas a diversos sectores económicos, y ya que tienen la posibilidad de generar valor agregado al producir bienes de manera sustentable.

El enfoque adoptado en este texto representa un aporte metodológico para la literatura de TS y sIT, ya que existe una necesidad de integrar métodos cuantitativos para el análisis de estos enfoques de pensamiento. La intención del artículo fue demostrar el estado de desarrollo del sit de UDEg en México. 


\section{BIBLIOGRAFÍA}

Agencia Internacional de Energías Renovables (IRENA) (2017). Acelerando la transformación energética mundial. REpensando La Energía 2017, 12.

Araújo, K. (2014). "The emerging field of energy transitions: progress, challenges, and opportunities". Energy Research and Social Science 1. https:// doi.org/10.1016/j.erss.2014.03.002.

Bento, N. y Fontes, M. (2015). The construction of a new technological innovation system in a follower country: Wind energy in Portugal. Technological Forecasting and Social Change, 99. https://doi.org/10.1016/j. techfore.2015.06.037

Bergek, A., Jacobsson, S., Carlsson, B., Lindmark, S. y Rickne, A. (2008). Analyzing the functional dynamics of technological innovation systems: A scheme of analysis. Research Policy, 37(3). https://doi.org/10.1016/j. respol.2007.12.003

Blei, D. M., Ng, A. Y. y Jordan, M. I. (2003). Latent dirichlet allocation. Journal of Machine Learning Research, 3(4-5). https://www.jmlr.org/papers/volume3/blei03a/blei03a.pdf

, Carin, L. y Dunson, D. (2012). Probabilistic topic models. IEEE Signal Processing Magazine, 27(6). https://doi.org/10.1109/MSP.2010.938079

Carlsson, B., Staffan, M. H. and Rickne A. (2002). "Innovation systems: analytical and methodological issues". Innovation Systems 31(2). https:// doi.org/10.1016/S0048-7333(01)00138-X.

Coenen, L. y Díaz López, F. J. (2010). Comparing systems approaches to innovation and technological change for sustainable and competitive economies: An explorative study into conceptual commonalities, differences and complementarities. Journal of Cleaner Production, 18(12). https://doi. org/10.1016/j.jclepro.2010.04.003

Díaz López, F. J. y Montalvo, C. (2015a). A comprehensive review of the evolving and cumulative nature of eco-innovation in the chemical industry. Journal of Cleaner Production, 102. https://doi.org/10.1016/j.jclepro. 2015.04.007

y Montalvo, C. (2015b). Qualitative data mining of large datasets: The case of long waves of eco-innovation in the chemical industry (19012011). TNO Working Paper Series, No. 2015-04. May, Delft.

Ding, Z., Li, Z. y Fan, C. (2018). Building energy savings: Analysis of research trends based on text mining. Automation in Construction, 96. https://doi. org/10.1016/j.autcon.2018.10.008 
Diario Oficial de la Federación (DOF) (2014). Ley de Energía Geotérmica Capítulo I Disposiciones Generales, 1-22. Retrieved from http://www. diputados.gob.mx/LeyesBiblio/pdf/LEG_110814.pdf

Edsand, H. E. (2019). Technological innovation system and the wider context: A framework for developing countries. Technology in Society, 58. https://doi.org/10.1016/j.techsoc.2019.101150

Grimmer, J. y Stewart, B. M. (2013). Text as data: The promise and pitfalls of automatic content analysis methods for political texts. Political Analysis, 21(3). https://doi.org/10.1093/pan/mps028

Hekkert, M. P., Suurs, R. A. A., Negro, S. O., Kuhlmann, S. y Smits, R. E. H. M. (2007). Functions of innovation systems: A new approach for analysing technological change. Technological Forecasting and Social Change, 74(4). https://doi.org/10.1016/j.techfore.2006.03.002

Hiriat, G. (2011). Evaluación de la Energía Geotérmica en México. Informe para el Banco Interamericano de Desarrollo y la Comisión Reguladora de Energía. Informe preparado para la CRE, con el apoyo del BID. InterAmerican Development Bank (IDB). https://www.cre.gob.mx//documento/2027.pdf

International Energy Agency (IEA) (2019). World Energy Outlook 2019. https://www.iea.org/reports/world-energy-outlook-2019

Jacobsson, S. y Bergek, A. (2004). Transforming the energy sector: The evolution of technological systems in renewable energy technology. Industrial and Corporate Change, 13(5). https://doi.org/10.1093/icc/dth032

Kiruja, J. (2011). Direct utilization of geothermal energy. Direct Utilization of Geothermal Energy, 3. https://orkustofnun.is/gogn/unu-gtp-sc/UNUGTP-SC-15-0904A.pdf

Líndal, B. (1992). Review of industrial applications of geothermal energy and future considerations. Geothermics, 21(5-6). https://doi.org/10.10 16/0375-6505(92)90012-X

Link, K., Rybach, L., Imhasly, S. y Wyss, R. (2015). Geothermal Energy in Switzerland-Country Update, Proceedings World Geothermal Congress 2020, Reykjavik, Iceland, April 26-May 2, 2020. https://geothermieschweiz.ch/wp_live/wp-content/uploads/2020/09/Link_et_al_2020_ Geothermal_Energy_in_Switzerland_2015-2020.pdf

Lund, J. W. y Boyd, T. L. (2015). Direct utilization of geothermal energy 2015 worldwide review. Proceedings World Geothermal Congress, Melbourne, Australia, 19-25 April 2015 (pp. 19-25). https://doi.org/10.3390/en3081443 
y Toth, A. N. (2020). Direct Utilization of Geothermal Energy 2020 Worldwide Review. Geothermics 60. https://doi.org/10.1016/j.geothermics.2015.11.004

Markard, J. y Truffer, B. (2008). Technological innovation systems and the multi-level perspective: Towards an integrated framework. Research Policy, 37(4). https://doi.org/10.1016/j.respol.2008.01.004

Mohr, J. W. y Bogdanov, P. (2013). Introduction-topic models: What they are and why they matter. Poetics, 41(6). https://doi.org/10.1016/j.poetic.2013.10.001

Morales, A. C., Pérez, M., Pérez, J. R. y Almaraz, S. (2017). Energías renovables y el hidrógeno: un par prometedor en la transición energética de México. Investigación y Ciencia de la Universidad Autónoma de Aguascalientes, (70). http://www.redalyc.org/articulo.oa?id=67451351012

North, D. (1990). Frontmatter. In institutions, institutional change and economic performance (Political economy of institutions and decisions, pp. i-iv). Cambridge University Press.

Nowlin, M. C. (2016). Modeling issue definitions using quantitative text analysis. Policy Studies Journal, 44(3). https://doi.org/10.1111/psj.12110

Perrot, R. (2015). The dynamics of renewable energy transition in developing countries: The case of South Africa and India. Working Paper Series No. \#2012-067. https://www.merit.unu.edu/publications/working-papers/abs tract $/$ ?id $=4803$

Ragnarsson, Á. (2003). Utilization of geothermal energy in Iceland. International Geothermal Conference. Reykjavík, Sept. http://citeseerx.ist.psu.edu/ viewdoc/download?doi=10.1.1.482.4123\&rep=rep1\&type=pdf

Romo-Jones, J. M., Gutiérrez-Negrin, L. C. y Canchola-Félix, I. (2020). 2019 Mexico Country Report, no. June.

Sandoval, L. (1998). La transición energética contemporánea. Problemas del Desarrollo. Revista Latinoamericana de Economia, 29(113). https://probdes.iiec.unam.mx/index.php/pde/article/view/28380

Santoyo, E., Almirudis-Echeverría, J. A. y Loya, W. (2012). Geotermia: energía de la tierra. Editorial Terracota, Colección Sello de Arena.

Santoyo-Castelazo, E., Stamford, L. y Azapagic, A. (2014). Environmental implications of decarbonising electricity supply in large economies: The case of Mexico. Energy conversion and management, 85. http://doi. org/10.1016/j.enconman.2014.05.051

Santoyo-Gutiérrez, E. y Torres-Alvarado, I. S. (2010). Escenario futuro de explotación de la energía geotérmica: hacia un desarrollo sustentable. Digital 
Universitaria, 11, 26. Retrieved from http://posgrado.ier.unam.mx/static/ admision/geotermia/Geotermia-guia-2.pdf

Sawulski, J., Gałczyński, M. y Zajdler, R. (2019). Technological innovation system analysis in a follower country-The case of offshore wind in Poland. Environmental Innovation and Societal Transitions, 33. https://doi. org/10.1016/j.eist.2019.07.002

Secretaría de Energía (sener) (2013). Prospectiva de Energías Renovables 2013-2027. México, sener, pp. V, 24, 25, 41, 50, 51, 61.

Silva, J. A. (2019). Renewable Energy in Mexico: Challenges and Opportunities. Espacios, 40(25). https://ipn.elsevierpure.com/en/publications/ renewable-energy-in-mexico-challenges-and-opportunities

Sircar, A., Kriti, Y. y Shreya, S. (2016). Overview on Direct Application of Geothermal Energy. International Advanced Research Journal in Science, Engineering and Technology, 3(9). https://iarjset.com/upload/2016/september-16/IARJSET\%2025.pdf

Suurs, R. (2009). Motors of sustainable innovation: Towards a theory On the Dynamics of Technological Innovation Systems. Utrecht University.

, Hekkert, M. P. (2009a). Cumulative causation in the formation of a technological innovation system: The case of biofuels in the Netherlands. Technological Forecasting and Social Change, 76(8). https://doi. org/10.1016/j.techfore.2009.03.002

, Hekkert, M. P., Kieboom, S. y Smits, R. E. H. M. (2009b). Understanding the formative stage of Technological Innovation System development. The case of natural gas as an automotive fuel, Innovation Studies Utrecht (ISU) working paper series 09-09, Utrecht University, Department of Innovation Studies, revised Jun 2009.

y Hekkert, M. (2012). Motors of sustainable innovation: Understanding transitions from a technological innovation system's perspective. Governing the Energy Transition: Reality, Illusion or Necessity? https://doi. org/10.4324/9780203126523

Tigabu, A. D., Berkhout, F. y van Beukering, P. (2015). Technology innovation systems and technology diffusion: Adoption of bio-digestion in an emerging innovation system in Rwanda. Technological Forecasting and Social Change, 90(PA). https://doi.org/10.1016/j.techfore.2013.10.011

Tomasini-Montenegro, C., Santoyo-Castelazo, E., Gujba, H., Romero, R. y Santoyo, E. (2017). Life cycle assessment of geothermal power generation technologies: An updated review. Applied Thermal Engineering, 114. https://doi.org/10.1016/j.applthermaleng.2016.10.074 
Van De Ven, A. H. y Poole, M. S. (2005). Alternative approaches for studying organizational change. Organization Studies, 26(9). https://doi. org/10.1177/0170840605056907

Wieczorek, A. J. (2018). Sustainability transitions in developing countries: Major insights and their implications for research and policy. Environmental Science and Policy, 84. https://doi.org/10.1016/j.envsci.2017.08.008

Yin, R. K. (2009). Estudio de caso cap. 2. Applied Social Research Methods. https://doi.org/10.1086/421629 
